\title{
Knockout of NGAL aggravates tubulointerstitial injury in a mouse model of diabetic nephropathy by enhancing oxidative stress and fibrosis
}

\author{
XIAOLI LIU ${ }^{1}$, XINCHENG ZHAO ${ }^{1}$, XIAOTING DUAN ${ }^{1}$, XIAOYING WANG ${ }^{1}$, TAOXIA WANG ${ }^{1}$, \\ SHUNING FENG $^{1}$, HUIFANG ZHANG ${ }^{1}$, CHENG CHEN $^{2}$ and GUIYING LI ${ }^{1}$ \\ Departments of ${ }^{1}$ Nephrology and ${ }^{2}$ Oncology, Affiliated Hospital of Hebei University of Engineering, \\ Handan, Hebei 056000, P.R. China
}

Received April 26, 2019; Accepted July 29, 2020

DOI: $10.3892 /$ etm.2021.9752

\begin{abstract}
Neutrophil gelatinase-associated lipocalin (NGAL), also called lipocalin 2 , is considered a promising biomarker for acute and chronic kidney injuries. Several studies have demonstrated that its levels increase in plasma and urine in diabetic nephropathy (DN), and its urine concentration increases upon kidney function deterioration. However, its role in DN progression remains unclear. The current study used in vitro gene expression knockdown in human proximal tubular cell line human kidney (HK)2 to investigate the role of NGAL in oxidation and extracellular matrix secretion under high-glucose (HG) incubation. In addition, type 1 diabetes was induced in vivo in knockout $\mathrm{NGAL}^{-/}$and wild-type mice in order to investigate role of NGAL in the progression of DN. The results demonstrated that NGAL knockdown in HK2 cells significantly increased oxidative stress under HG stimulation tested by flow cytometry, and increased the secretion of interleukin-6, fibronectin (FN) and collagen IV examined by ELISA. Western blotting demonstrated that the phosphorylation of Smad2/3 also increased in HK2 cells under transforming growth factor- $\beta 1$ stimulation. In vivo experiments demonstrated that diabetic $\mathrm{NGAL}^{-/-}$mice showed deteriorated renal function compared with that of diabetic wild-type mice. Histopathological analysis suggests that diabetic $\mathrm{NGAL}^{-/}$mice had more serious glomerulosclerosis and tubular vascular degeneration than wild-type mice. Immunohistochemistry suggested that the absence of NGAL lead to increased FN deposition in glomeruli in a mouse model of DN. In conclusion, NGAL appears to have renal protective effects by slowing
\end{abstract}

Correspondence to: Dr Guiying Li, Department of Nephrology, Affiliated Hospital of Hebei University of Engineering, 81 Cong Tai Road, Handan, Hebei 056000, P.R. China

E-mail: 55435660@qq.com

Key words: neutrophil gelatinase-associated lipocalin, diabetic nephropathy, histopathological analysis, fibrosis, knockdown, knockout down the progression of $\mathrm{DN}$, and its effect may be associated with a reduction in oxidation, fibrosis and inflammation.

\section{Introduction}

Diabetes mellitus is a prevalent autoimmune disease worldwide that can develop at any stage of life, including adulthood and childhood. Diabetic nephropathy (DN) is a chronic and severe complication associated with increased risk of end-stage renal failure and cardiovascular disease (1). Evidence suggests that $>30 \%$ of all patients with diabetes develop DN within 10-20 years of the onset of diabetes $(2,3)$. In patients with diabetes, the incidence of development of microalbuminuria is $20-40 \%$ if DN remains untreated. Moreover, within 20-25 years, almost $20 \%$ of these patients will develop end-stage renal failure (4) and further require renal transplantation or chronic hemodialysis (5). Effective medications for DN and anti-hypertension drugs are still the first-line choice; therefore, identifying new drug targets and new drug candidates is urgently required $(6,7)$.

Neutrophil gelatinase-associated lipocalin (NGAL) is a biomarker of renal tubular injury that is upregulated in distal tubules and the collecting duct, and its role in acute kidney injury (AKI) has been extensively evaluated $(8,9)$. NGAL is a $25-\mathrm{kDa}$ glycoprotein with 178 amino acids that belongs to the lipocalin superfamily (10). It is a constituent of specific granules and exists in neutrophils as part of the NGAL-gelatinase complex (11). It is involved in the antimicrobial defense mechanism and is upregulated in systemic bacterial infections $(12,13)$. It also plays a protective role in epithelial injury due to its antiapoptotic effect (14). As it is not produced by necrotic nephron, it is a marker of active injury and represents the mass of salvageable nephrons (15). Its usefulness as a biomarker in chronic kidney diseases $(16,17)$ such as DN has been reported by several previous studies, however, its role and effect in the pathophysiology of DN progression remains unclear. In the current study both in vitro and in vivo methods were used. NGAL was knocked down in human tubular epithelial cells and NGAL knockdown mice were used in order to investigate the exact role of NGAL in DN. 


\section{Materials and methods}

Cell culture and NGAL knockdown. Human kidney (HK)2, an immortalized human proximal tubular cell line, was purchased from the American Type Culture Collection. HK2 cells were cultured in DMEM (Invitrogen; Thermo Fisher Scientific, Inc.) supplemented with $10 \%$ fetal bovine serum (HyClone; GE Healthcare Life Sciences) and passaged $<10$ times. The cells were supplemented with $2 \mathrm{mmol} / 1$ glutamine, $100 \mathrm{U} / \mathrm{ml}$ penicillin and $100 \mathrm{U} / \mathrm{ml}$ streptomycin, and maintained in a $37^{\circ} \mathrm{C}, 5 \% \mathrm{CO}_{2}$ humidified atmosphere. The culture medium was changed every 2-3 days.

To knock down NGAL expression, a lentivirus pHBLV-U6-Zsgreen harboring a short hairpin RNA (shRNA) of NGAL synthesized by Gene Company, Ltd. was used, as previously described by the supplier. The NGAL shRNA sequence was 5'-GGACTTTTGTTCCAGGTTGTTAACAAC CTGGAACAAAAGTCC-3'. A period of one day before the transfection, HK-2 cells were seeded into a six-well plate. When $\sim 80 \%$ confluency of cells was reached, cells were transfected. The confluency was calculated as the area of cells/the whole view under an optical microscope (BM-37XBC, Shanghai BM optical instruments manufacture Co., Ltd.) at a magnification of x100. Transfection was performed with $1.2 \mu \mathrm{g}$ shRNA plasmid containing NGAL (cat. no. sc-43969-V; Santa Cruz Biotechnology, Inc.) and $4.5 \mu \mathrm{l}$ HiPerFect transfection reagent (Qiagen China Co., Ltd.) were added into $100 \mu 1$ DMEM without FBS. A period of 15 min later, the aforementioned mixture $(5 \mu \mathrm{l})$ was dropped into wells $(2 \mathrm{ml} /$ well), DMEM was replaced with complete culture medium (DMEM+10\% FBS) $24 \mathrm{~h}$ later. After incubation at $37^{\circ} \mathrm{C}$ for one day, cells were split 1:5. After another day of culture at $37^{\circ} \mathrm{C}$, stable clones expression the ShRNA were selected using $10 \mu \mathrm{g} / \mathrm{ml}$ puromucin dihydrochloride (cat. no. P8230; Beijing Solarbio Science \& Technology Co., Ltd.). The medium containing puromucin selection was replaced every 3 days and identified the resistant colonies. Once a cell line was generated, the knockdown efficiency was determined using reverse transcription-quantitative (RT-q)PCR (18). A non-targeting shRNA was also prepared and transfected into HK2 cells as a negative control (NC). Cells transfected with the NC lentivirus were termed NC-HK2, while NGAL knockdown cells were called KD-HK2.

mRNA quantification by $R T-q P C R$. Total RNA was extracted using the TRNzol-A+ reagent kit (cat. no. DP430; Tiangen Biotech Co., Ltd.). RT-qPCR was performed with complementary DNA reverse transcribed from total RNA using a reverse transcription kit (ReverTra Ace ${ }^{\circledR}$ qPCR RT kit; Toyobo Life Science) and ABI PRISM ${ }^{\mathrm{TM}} 7000$ (Applied Biosystems; Thermo Fisher Scientific, Inc.). The denaturation and annealing reaction was conducted at $65^{\circ} \mathrm{C}$ for $5 \mathrm{~min}$ and $4^{\circ} \mathrm{C}$. The reverse transcription was performed at $45^{\circ} \mathrm{C}$ for $20 \mathrm{~min}, 95^{\circ} \mathrm{C}$ for $5 \mathrm{~min}$ and $4^{\circ} \mathrm{C}$ for $5 \mathrm{~min}$. SYBR green was used as the fluorophore and $\beta$-actin was used as an internal control. The forward and reverse primers for $\beta$-actin were 5'-AAACAGAAGGCAGCT TTACGATG-3' and 5'-AAATGTTCTGATCCAGTAGCG-3', respectively. For NGAL, the forward primer was 5'-TCCCAG AGCTGAACGG-3' and the reverse primer was 5'-GAAGTC GCGGAGACA-3'. The qPCR thermocycling conditions were $95^{\circ} \mathrm{C}$ for $30 \mathrm{sec}$, followed by 40 cycles of $95^{\circ} \mathrm{C}$ for $15 \mathrm{sec}$, $60^{\circ} \mathrm{C}$ for $30 \mathrm{sec}$ and $72^{\circ} \mathrm{C}$ for $30 \mathrm{sec}$. The $2^{-\Delta \Delta C \mathrm{Cq}}$ value was normalized to the signal of the housekeeping gene, $\beta$-actin. The fold-change in expression was calculated as the method described previously (19).

Stimulation by normal (NG) and high glucose (HG). Upon reaching $60-80 \%$ confluence, NC-HK2 and KD-HK2 cells were growth-arrested in $0.5 \%$ fetal calf serum (HyClone; GE Healthcare Life Sciences) for $48 \mathrm{~h}$ and treated with 5 (as normal glucose) and $25 \mathrm{~mm}$ (as high glucose) D-glucose for $\leq 48 \mathrm{~h}$.

ELISA for collagen IV (Col IV), fibronectin (FN) and interleukin (IL)- 6 secretion by HK2 cells under HG stimulation. Exposure of $\mathrm{HK} 2$ cells to medium containing high concentrations of glucose induced the overproduction of FN, Col IV and IL-6, as described in a previous study (20). To determine the effect of NGAL on the increased expression of FN, Col IV and IL- 6 triggered by HG, NC-HK2 and KD-HK2 cells were treated with HG for $48 \mathrm{~h}$. The levels of FN, Col IV and IL-6 in the supernatant were measured with ELISA kits for FN (cat. no. ab219046; Abcam), IL-6 (cat. no. ab178013; Abcam) and Col IV (Elabscience). The concentration in the culture supernatant was normalized to the total protein concentration in the cells, as quantified by the BCA method.

Analysis of intracellular reactive oxygen species (ROS) production. The intracellular formation of ROS was detected by incubating cultured HK2 cells with $1 \mu \mathrm{m}$ of the 2',7'-dichlorofluorescin diacetate (DCFHA; cat. no. D6883; Merck), a nonpolar compound that is converted into the nonfluorescent polar derivative 2',7'-dichlorofluorescin (DCFH) by cellular esterase upon incorporation into the cells. DCFH is then oxidized to the highly fluorescent 2',7'-dichlorofluorescein (DCF) in the presence of oxidants. Briefly, HK2 cells were incubated for $30 \mathrm{~min}$ at $37^{\circ} \mathrm{C}$ with $1 \mu \mathrm{m} \mathrm{H}$ 2DCFDA in normal glucose (NG) or HG $(25 \mathrm{~mm})$ conditions. The fluorescence intensity was determined immediately with a flow cytometer [excitation wavelength $(\lambda)=488 \mathrm{~nm}$, emission $\lambda=515 \mathrm{~nm}$; BD FACSCalibur (BD Biosciences); Cell Quest Pro software (version 5.1; BD Biosciences)]. The average fluorescence intensity was normalized to the total cell protein content in each group, as described previously $(21,22)$.

Generation of $\mathrm{NGAL}^{-/-}$mice. NGAL ${ }^{-1-} \mathrm{C} 57 \mathrm{BL} / 6$ mice were generated by Cyagen Biosciences, Inc. The strategy of NGAL knockout was as follows: A genomic DNA clone containing exons 1-6 of the murine NGAL gene was isolated from a 129/J mouse genomic library (Cyagen Biosciences, Inc.). A target vector was designed to replace a $2.5-\mathrm{kb}$ genomic fragment containing NGAL2 exons 1-5 with the PGK-neo cassette. The diphtheria toxin A gene driven by the pMC1 promoter was incorporated into the 3 ' end of the vector for negative selection. The targeting vector was linearized with NotI and electroporated into 129/Ola ES cells using a Bio-Rad Gene Pulser at $0.34 \mathrm{kV}$ and $250 \mu \mathrm{F}$ (Bio-Rad Laboratories, Inc.). According to manufacturers protocol, $400 \mu \mathrm{l}$ cells $\left(2 \times 10^{6}\right.$ cells $\left./ \mathrm{ml}\right)$ and $20 \mu \mathrm{g}$ targeting vector in the Opti-MEM (Thermo Fisher Scientific, Inc.) were mixed in a $0.4 \mathrm{~cm}$ cuvette, which was chilled in ice. The electroporation was conducted at $4^{\circ} \mathrm{C}$ and with twice pulse 
immediately after the signal, one pulse lasting for $2 \mathrm{sec}$, and one pulse lasting for $3 \mathrm{sec}$. After electroporation, the medium was replaced with growth medium (DMEM+10\% FBS as aforementioned). The transfected cells were then cultured in growth medium at $20^{\circ} \mathrm{C}$ overnight and then combined with $300 \mu \mathrm{g} / \mathrm{ml}$ Geneticin (G418; Sigma-Aldrich; Merck KGaA) for 10 days. Homologous recombinants were identified by PCR and verified by Southern blotting with a PCR-generated 5 ' flanking probe (forward and reverse primers, 5'-ATAGCCCTGGCTGTC CTGAA-3' and 5'-TAAGGTCCCCCTCTAAACC-3', respectively). Correctly targeted clones were injected into C57BL/6J blastocysts, and the generation of chimeras was performed. A total of two independently targeted ES cell clones transmitted the NGAL mutation into the germ line. Male chimeric mice were mated to C57BL/6 female mice, and the heterozygous F1 progeny was intercrossed to generate NGAL-deficient mice. The wild-type littermates from these crosses were used as controls for all experiments. All mice were maintained in the animal facility of the Affiliated Hospital of Hebei University of Engineering in accordance with established ethical regulations of animal care.

Induction and assessment of diabetes. Adult wild-type male C57 BL/6 mice (18-20 g) were purchased from Beijing Weitong Lihua Experimental Animal Technology Co., Ltd. The animals were maintained at $24^{\circ} \mathrm{C}$ with a relative humidity of $45-55 \%$ and $12 / 12 \mathrm{~h}$ dark/light cycle, and had free access to food and water throughout the experimental protocol.

Both wild-type C57 BL/6 (4 male and 4 female mice; body weight: $24 \pm 2 \mathrm{~g}$; age: 6 weeks) and NGAL ${ }^{-/-} \mathrm{C} 57 \mathrm{BL} / 6$ mice ( 4 male and 4 female mice, body weight: $24 \pm 2 \mathrm{~g}$; age: 6 weeks) were used to establish an experimental model of DN. A single dose of $60 \mathrm{mg} / \mathrm{kg}$ streptozotocin (STZ; cat. no. S0130; Merck KGaA) prepared in citrate buffer ( $\mathrm{pH} 4.4 ; 0.1 \mathrm{M})$ was injected intraperitoneally to induce diabetes. C57 BL/6 mice who received PBS injection were considered as the normal control group. Diabetes was confirmed 4 days after STZ injection. Blood samples were collected via the retro-orbital plexus using heparinized capillary glass tubes and plasma glucose levels were estimated with an enzymatic glucose oxidase-peroxidase diagnostic kit (Span Divergent Ltd). Animals were anesthetized prior to sample collection. Mice with plasma glucose levels $>250 \mathrm{mg} / \mathrm{dl}$ were selected and used in the present study (for each group there were 8 mice). Wild-type C57 BL/6 mice with DN were named as DN-wild, and NGAL ${ }^{-/}$C57 BL/6 mice with DNA were named as $\mathrm{DN}-\mathrm{NGAL}^{-/}$. Healthy mice were named as the normal control group. Their body weight and plasma glucose levels were measured before and at the end of the experiment. The animal health and behavior were monitored every day, and no animals died during the experiment. All animal welfare considerations were taken, including efforts to minimize suffering and distress. Based on literature and a preliminary study, after three months of STZ-induced diabetes, the mice progress into DN with proteinuria and glomerulosclerosis; and reaching the humane endpoint of $20 \%$ weight-loss was considered a humane endpoint (23). After 3 months, all mice were euthanatized by cervical dislocation, and pupillary response to light was used to confirm the death of animals. Briefly, a bright light was shone into the eyes of the animals.
A constriction of the pupil indicated a neurological response. Upon death, the pupils become dilated and unresponsive to light. Kidney tissue blocks were collected and fixed in $10 \%$ formalin at $4^{\circ} \mathrm{C}$ for $24 \mathrm{~h}$, then the samples were ready for histological examination and immunohistochemistry (IHC).

The experimental protocol was approved by the Institutional Animal Ethics Committee of the Affiliated Hospital of Hebei University of Engineering (Handan, China) and performed in accordance with the guidelines on animal experimentation of the Committee for Control and Supervision of Experimentation on Animals, Government of China (24).

Renal function. Every 4 weeks, blood was sampled through the eyes under anesthesia with sodium pentobarbital solution (60 mg/kg; intraperitoneal injection). Blood urea nitrogen (BUN) and serum creatinine (Scr) was measured using an automated biochemical analyzer (200 FR; Toshiba Corporation). Urine was collected, the urine albumin content was measured using the mouse albumin ELISA kit (cat. no. ab108792; Abcam), and the urine creatinine was measured using the automated biochemical analyzer. Albuminuria was calculated as the ratio of urine albumin divided by urine creatinine.

Histological examination. Fixed renal tissue blocks were embedded in paraffin. Sections of $2-\mu \mathrm{m}$ thickness were cut and stained with hematoxylin for $10 \mathrm{~min}$ and then eosin for $5 \mathrm{~min}$ at room temperature and periodic acid-Schiff for $10 \mathrm{~min}$ at room temperature. The stained sections were examined under a light microscope (magnification, x200). The severity of glomerulosclerosis was graded in a blinded manner on a scale of 0-4 as previously described (25): Grade 0, normal; grade 1 , sclerotic area $\leq 25 \%$ (minimal); grade 2 , sclerotic area $>25-50 \%$ (moderate); grade 3 , sclerotic area $>50-75 \%$ (moderate to severe); and grade 4 , sclerotic area $>75-100 \%$ (severe). The scores from each individual glomerulus examined (100 glomeruli from each animal) were averaged, and the percentage of glomeruli for each grade was calculated.

Tubular atrophy, dilation, casts, interstitial inflammation and fibrosis were the major pathological characteristics of renal interstitium. A total of 10 microscopic fields from each animal (magnification, x200) were selected for quantitation based on a previously established scoring system $(26,27)$ : 0 , normal; 1 , lesions in $<25 \%$ of the area; 2 , lesions in $25-50 \%$ of the area; 3 , lesions in $>50 \%$ of the area; and 4 , lesions involving the entire area.

Electron microscopy. To further investigate the effect of NGAL on renal ultrastructure, transmission electron microscopy (TEM) was conducted. Ultra-thin sections $(2 \mu \mathrm{m})$ were prepared according to the method previously described (28). Briefly, a small piece of the left kidney (1x1 mm) was fixed in $2.5 \%$ glutaraldehyde solution buffered with sodium cacodylate at $\mathrm{pH} 7.4$ for $2 \mathrm{~h}$; post-fixed for $1 \mathrm{~h}$ in $1 \%$ osmium tetroxide solution at $25^{\circ} \mathrm{C}$ and $\mathrm{pH} 7.4$; and rehydrated in ethanol. Upon embedding, sections were stained with uranyl acetate and lead citrate. TEM was performed with a JEM-1400Flash electron microscope (JEOL, Ltd.) for viewing and image capture. Evaluations were performed by two independent observers in a blinded manner. Grading for the presence of changes in the podocyte foot processes was analysed according to the 
following scale: 0 , no evidence of changes; I, $<25 \%$ changes; II, $25-50 \%$ changes; III, $>50-75 \%$ changes; and IV, $>75 \%$ changes. Measurements were obtained from electronographs (magnification, x10,000) $(29,30)$.

IHC analysis. IHC was used to investigate the expression of NGAL and FN. Rabbit monoclonal antibodies against NGAL (cat. no. ab216462) and FN (cat. no. ab268020) were purchased from Abcam. Paraffin-embedded sections were dewaxed by xylene, and endogenous peroxidase activity was quenched with $3 \% \mathrm{H}_{2} \mathrm{O}_{2}$. Sections were digested with proteinase $\mathrm{K}$ for antigen retrieval. 2\% BSA (cat. no. A2058; Merck KGaA) was used as the blocking reagent and incubated with the samples at room temperature for $30 \mathrm{~min}$. Sections were incubated with primary antibodies $(1: 100)$ overnight at $4{ }^{\circ} \mathrm{C}$ and peroxidase conjugated goat anti-rabbit IgG (1:200, TA140003; OriGene Technologies, Inc.) at $4^{\circ} \mathrm{C}$ for $30 \mathrm{~min}$. IHC staining-positive areas were counted in 30 random cortex $x 400$ high power fields (HPFs) under an Olympus microscope (IXplore; Olympus Corporation). Positive areas were measured using Image Pro 6.0 software (Media Cybernetics, Inc.) and expressed as percentage of positive areas per HPF.

Western blot analyses for transforming growth factor (TGF)-B1-mediated Smad3 and Smad2 phosphorylation. Human recombinant TGF- $\beta 1$ (cat. no. $240-B$; R\&D Systems, Inc.) was dissolved in deionized water supplemented with $0.1 \%$ BSA and $4 \mu \mathrm{m}$ hydrochloric acid. After serum starving for $24 \mathrm{~h}, 0.5 \mathrm{ng} / \mathrm{ml}$ TGF- $\beta 1$ was added to HK 2 cells $\left(5 \times 10^{5}\right.$ cells $\left./ \mathrm{ml}\right)$ at $37^{\circ} \mathrm{C}$ for $24 \mathrm{~h}$. HK2 cells without TGF- $\beta 1$ incubation were set as control. Then, the HK2 cells were harvested and lysed using RIPA buffer (Applygen Technologies, Inc.). Protein concentration was determined using the BCA method. A total of $50 \mu \mathrm{g}$ denatured protein per lane was separated using 12\% SDS-PAGE followed by the transfer onto PVDF membranes (Bio-Rad Laboratories, Inc.) as described in an established procedure $(31,32)$. The primary antibodies used (all, 1:100) were as follows: Anti-phosphorylated (p)-Smad2 (cat. no. 3108S); anti-p-Smad3 (cat. no. 9520S); anti-Smad2 (cat. no. 5339S); and anti-Smad3 (cat. no. 9523S; Cell Signaling Technology, Inc.). Samples were incubated with the PVDF membranes at $4^{\circ} \mathrm{C}$ overnight. The antibody for the reference $\beta$-actin was also purchased from Cell Signaling Technology, Inc (cat. no. 3108S). The secondary antibody anti-rabbit IgG (1:1,000; cat. no. 7074S; Cell Signaling Technology, Inc.) was incubated with the membranes at room temperature for $1 \mathrm{~h}$. Quantitation analysis of relative protein expression was performed using ImageJ 1. 53a (National Institutes of Health).

Western blot analyses for NGAL in NC-HK2 and KD-HK2 cells. This procedure was similar to that aforementioned. NC-HK2 and KD-HK2 cells were harvested and lysed using RIPA buffer (Beijing Solarbio Science \& Technology Co., Ltd). The primary antibody was LCN (D4M8L) rabbit monoclonal antibody (1:100; cat. no. 44058S; Cell Signaling Technology, Inc.), and the secondary antibody was the same as mentioned above.

MDA, GSH, SOD and catalase assay. The content of MDA, GSH, SOD and catalase was measured using MDA content

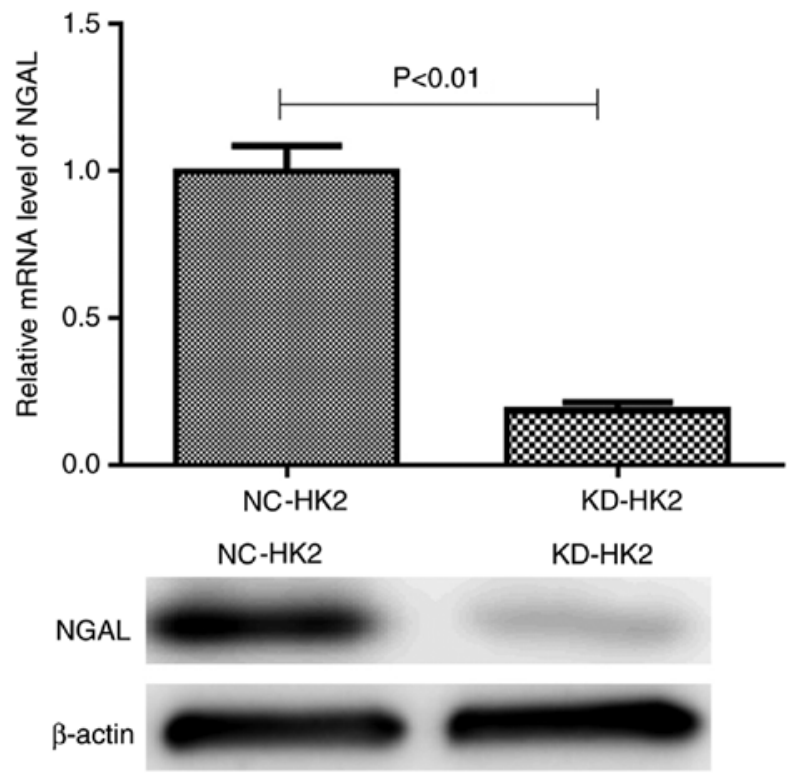

Figure 1. mRNA and protein levels of NGAL in NC-HK2 cells and NGAL KD-HK2 cells. NGAL, neutrophil gelatinase-associated lipocalin; HK2, human kidney 2; NC, negative control; KD, knockdown.

assay kit, GSH content assay kit, SOD content assay kit, and CAT content assay kit (cat. nos. BC0025, BC1175, BC0170, BC0200; Beijing Solarbio Science \& Technology Co., Ltd.) with the protocols provided from the supplier.

Statistical analysis. All values are expressed as the mean \pm standard deviation. SPSS 25.0 (IBM Corp.) was used for statistical analysis. The differences between two groups were evaluated using Student's t-test. Comparison of the same parameters among more than two groups was performed using one-way ANOVA, followed by post-hoc Tukey's test. $\mathrm{P}<0.05$ was considered to indicate a statistically significant difference.

\section{Results}

Efficiency of NGAL knockdown in HK2 cells. Both RT-qPCR and western blotting demonstrated that the mRNA and protein levels of NGAL in KD-HK2 cells were significantly reduced compared with those in NC-HK2 cells, which suggested that the knockdown efficiency was satisfactory for further experiments (Fig. 1).

NGAL knockdown enhances oxidative stress under hyperglycemia in vitro and in vivo. HG stimulation can induce intracellular oxidative stress in HK2 cells. Compared with that in NC-HK2 cells, KD-HK2 cells produced higher intracellular ROS levels under HG stimulation, which was demonstrated by flow cytometry (Fig. 2A and B). In addition, 3 months after the establishment of diabetes in animals, fresh kidney homogenate from diabetic mice was used to evaluate its oxidation status. As represented in Fig. 2C-F, compared with the findings in normal control animals, 3-months of diabetes led to higher malonaldehyde (MDA) and lower glutathione (GSH) levels, and lower activity of superoxide dismutase (SOD) and catalase (CAT) in kidney tissues. Furthermore, $\mathrm{NGAL}^{-/}$mice 

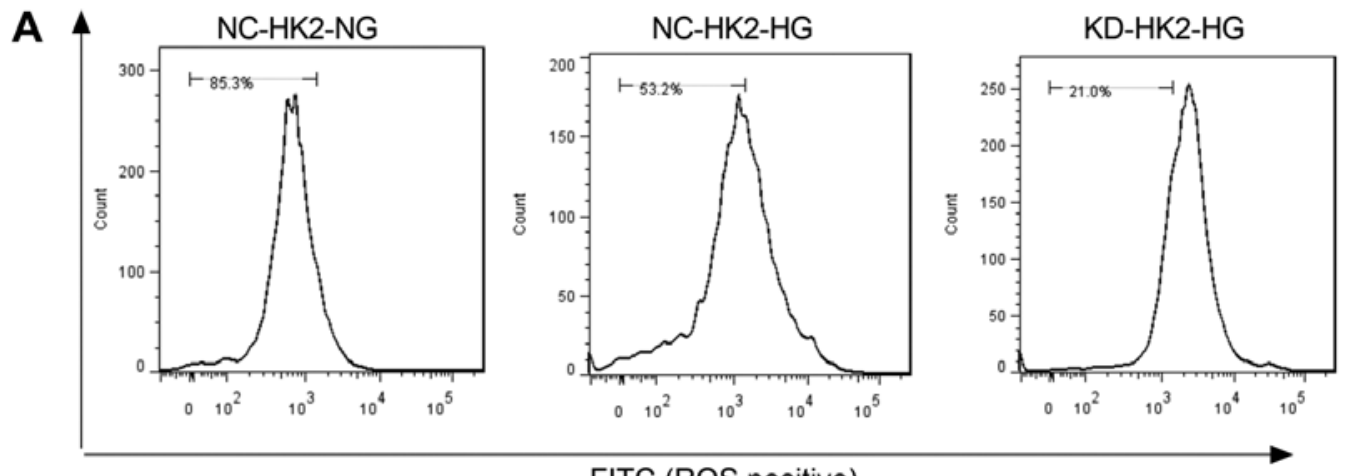

B

FITC (ROS positive)
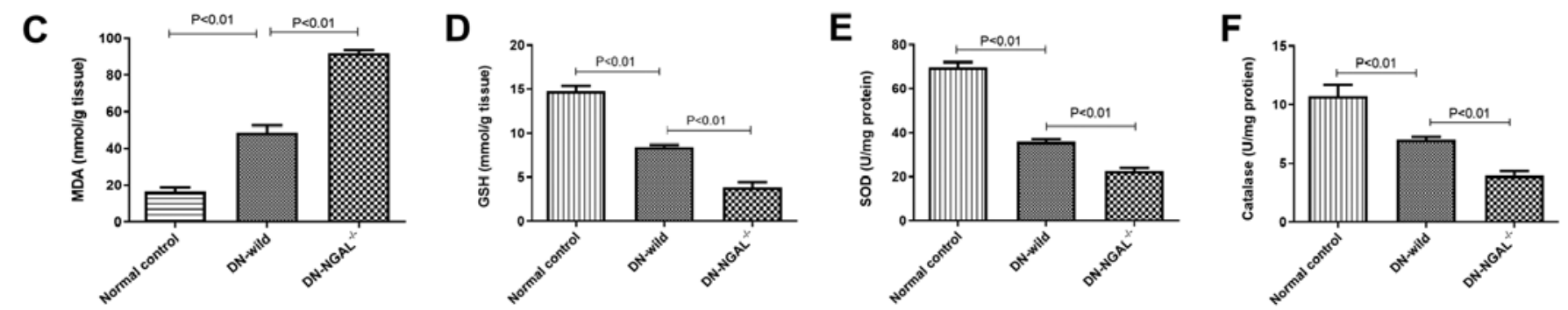

Figure 2. Oxidative stress measurement under glycemia in vitro and in vivo. (A) ROS test by flow cytometry in HK2 cells in vitro. (B) Quantitation of ROS levels in HK2 cells. (C) MDA, (D) GSH, (E) SOD and (F) catalase concentration or IU in kidney tissues in normal control, DN-wild and DN NGAL ${ }^{-/}$mice in vivo. NGAL, neutrophil gelatinase-associated lipocalin; HK2, human kidney 2; ROS, reactive oxygen species; MDA, malonaldehyde; GSH, glutathione; SOD, superoxide dismutase; DN, diabetic nephropathy; KD, knockdown; NC, negative control; HG, high glucose; NG, normal glucose.

showed higher oxidative stress in kidney tissues compared with that of normal control, with higher MDA and lower GSH levels, and lower activity of SOD and CAT in kidney tissues (Fig. 2C-F).

$N G A L$ knockdown in $H K 2$ cells reduces the secretion of $F N$, Col IV and IL-6 under HG conditions. HG stimulation for $48 \mathrm{~h}$ could significantly increase the secretion of FN, Col IV and IL-6 on HK2 cells in vitro, as measured by ELISA, compared with that of normal-glucose cultured cells (NG-NC-HK2 and NG-KD-HK2). This effect was shown in both HG-NC-HK2 and HG-KD-HK2 cells (Fig. 3). Compared with that of HG-NC-HK2 cells, the concentration of FN, Col IV and IL-6 in the supernatant of HG-KD-HK2 cell culture medium was significantly higher.

NGAL knockdown in HK2 cells enhances the phosphorylation of Smad2/3 by TGF- $\beta 1$ stimulation. TGF- $\beta 1$ is a key pro-fibrotic mediator, mainly via phosphorylation of $S m a d 2 / 3$. Our preliminary study suggested that low dosage $(0.5 \mathrm{ng} / \mathrm{ml})$ of TGF- $\beta 1$ stimulation could produce moderate phosphorylation of Smad2/3. Therefore, $0.5 \mathrm{ng} / \mathrm{ml} \mathrm{TGF-} \beta 1$ was used in the current study. As shown in Fig. 4A and 4B, under the same concentration of TGF- $\beta 1$, the protein level of $p-S m a d 2 / 3$ in high glucose treated KD-HK2 cells was significantly higher than that in NC-HK2 cells treated with either normal or high glucose. However, the total protein content of Smad2/3 did not change markedly among the three groups.

$N G A L^{-1-}$ mice show rapid deterioration of renal function and serious glomerular and tubular injury upon the establishment of diabetes. At 4 months, the BUN and Scr levels were measured. Compared with that exhibited by normal-control and DN-wild mice, DN-NGAL ${ }^{-1-}$ mice showed deterioration of renal function with higher BUN and Scr levels, and albuminuria (Fig. 5A-C), but no significant difference in fasting blood glucose was found between wild-type and $\mathrm{NGAL}^{-1-}$ mice. By histopathological analysis, the tubular injuries were noted to be more serious in $\mathrm{NGAL}^{-/-}$mice compared with those in wild-type mice, which had severe vacuolar degeneration in proximal tubules. By analyzing the glomerular morphology, $\mathrm{NGAL}^{-/}$mice were also observed to have serious glomerular sclerosis, glomerular hypertrophy, segmental and diffuse mesangial expansion compared with the glomerular morphology of wild-type mice (Fig. 5E-G). In order to further understand the ultrastructural alterations, TEM was performed to focus on the morphological changes of podocytes. As shown in Fig. 5H and I, the glomeruli from normal control animals had clear and orderly arranged foot processes; however, animals with DN exhibited severe podocyte foot process effacement. Compared with that of wild-type control mice, $\mathrm{NGAL}^{-1}$ mice showed more serious foot process effacement, which partially explained their higher albuminuria.

As shown in Fig. 6A, IHC confirmed that knockout $\mathrm{NGAL}^{-1-}$ mice did not express NGAL in kidney tissues, while normal mice showed light staining of NGAL and diabetic animals showed strong NGAL staining in the kidney cortex, mainly around tubular epithelial cells. FN staining was consistent with the in vitro results, and glycemia induced higher FN levels in the glomeruli, and DN-NGAL ${ }^{-1}$ mice exhibited higher FN deposit compared with that in DN-wild group (Fig. 6B). 

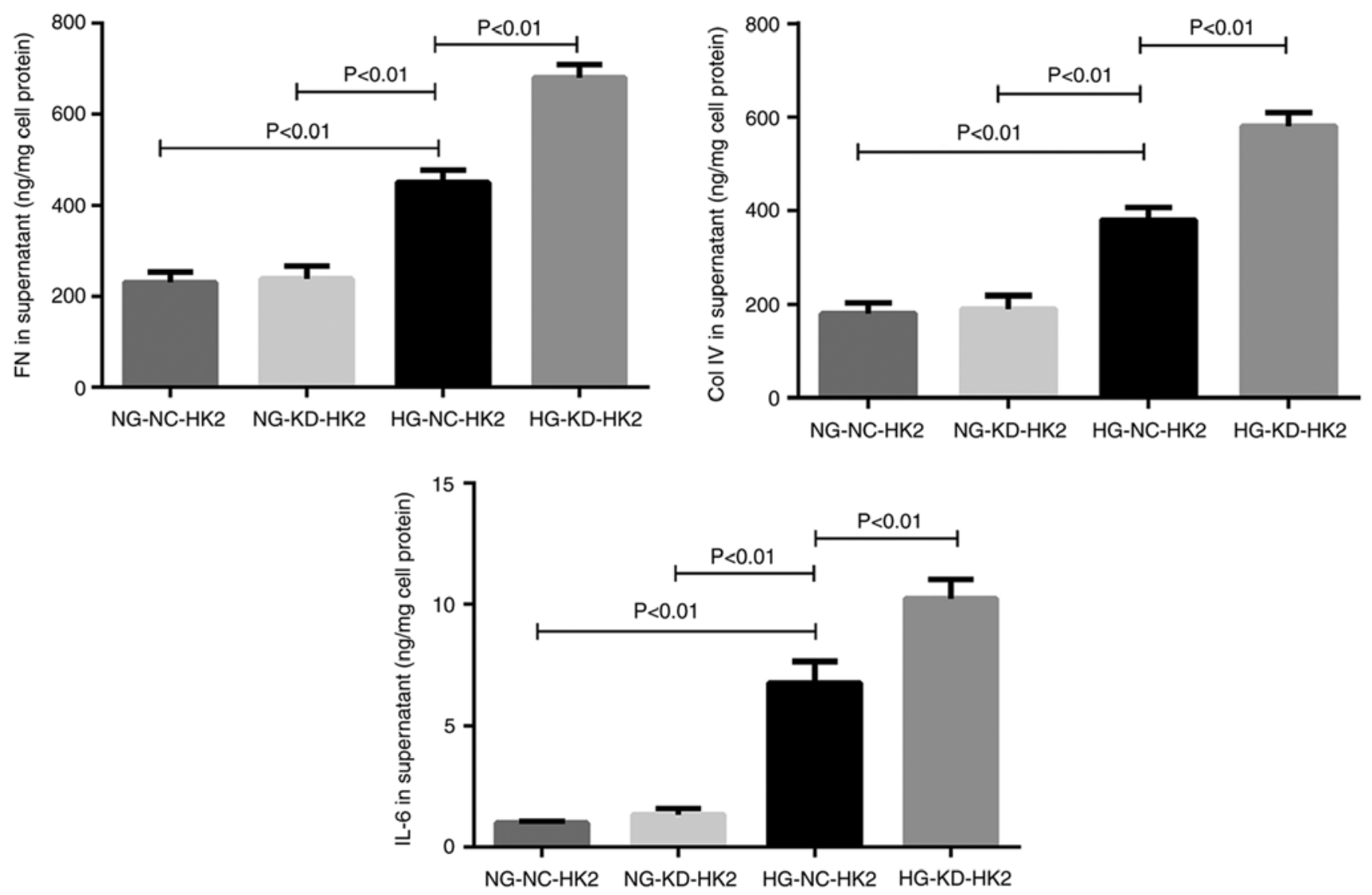

Figure 3. Concentration of FN, Col IV and IL-6 in the supernatant of HK2 cells stimulated by HG, as measured by ELISA. FN, fibronectin; Col IV, collagen IV; IL-, interleukin; HK2, human kidney 2; HG, high glucose; NG, normal glucose; NC, negative control; KD, knockdown.

A

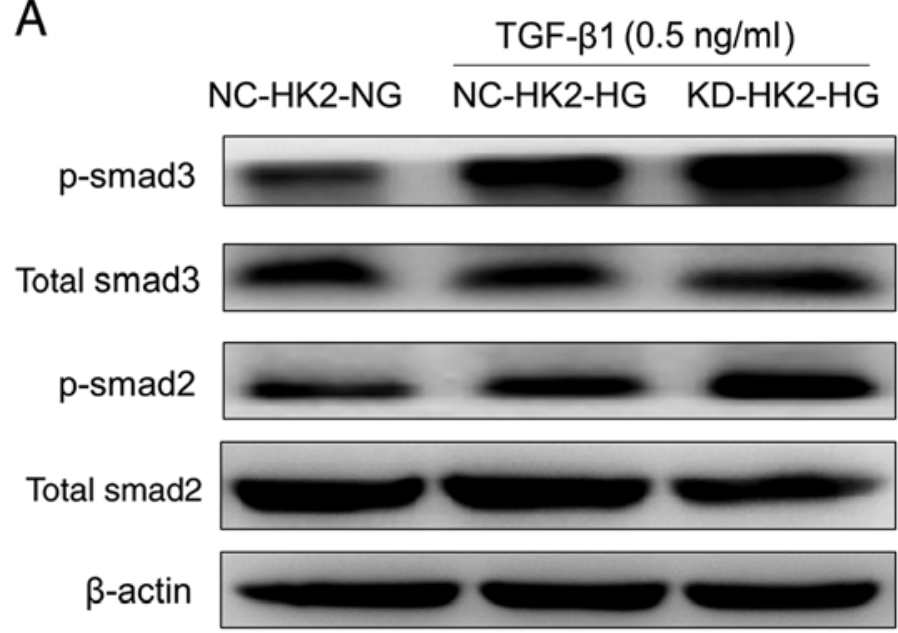

$\mathrm{B}$
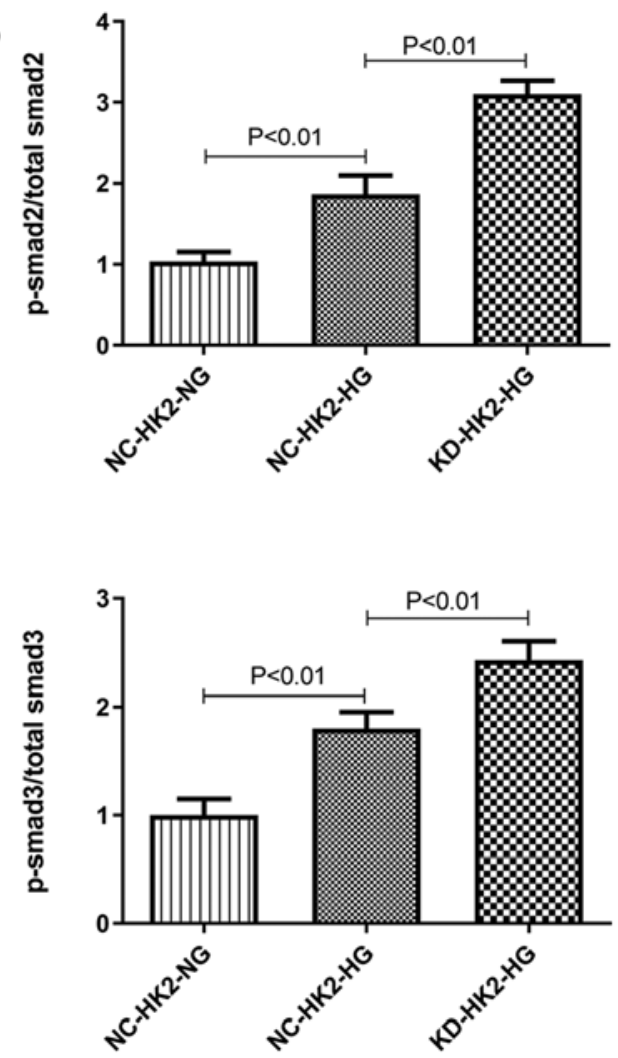

Figure 4. Protein level of total and p-Smad2/3 in human kidney 2 cells induced by TGF- $\beta 1$, as determined by western blotting. (A) Representative pictures of western blotting; (B) quantitation analysis of relative protein expression. p-, phosphorylated; TGF, transforming growth factor; NC, negative control; HK2, human kidney 2; LG, low glucose; HG, high glucose; KD, knockdown. 
A

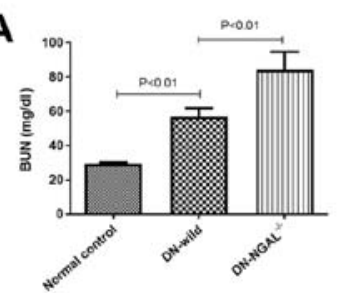

E

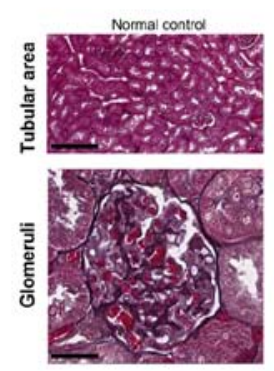

H

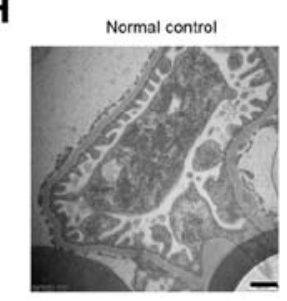

B

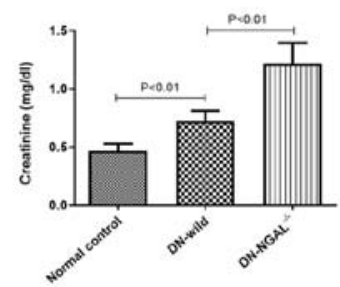

Glycemia
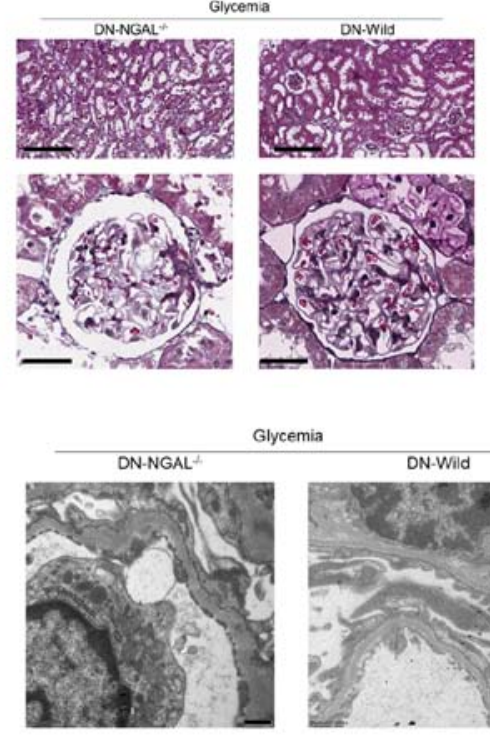

C

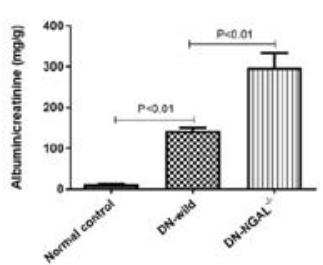

$\mathbf{F}$

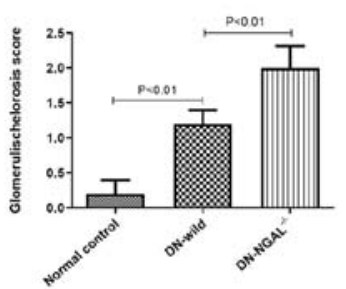

D

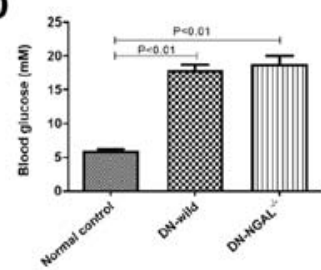

G

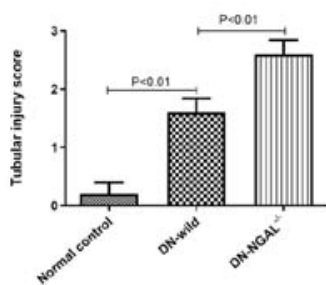

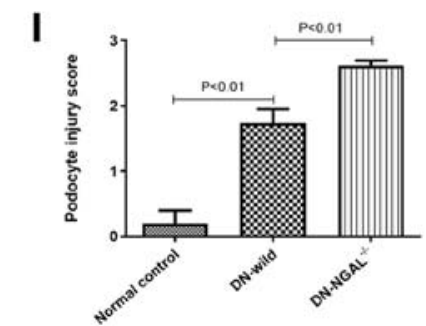

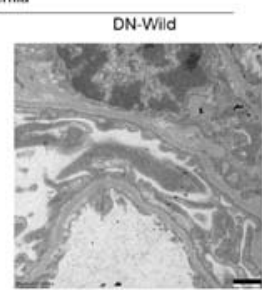

Figure 5. NGAL knockout exacerbates DN in mice. (A) BUN levels, (B) serum creatinine levels, (C) albuminuria and (D) fasting blood glucose concentration in the three animal groups. (E) Representative figures of H\&E and periodic acid-Schiff staining for histopathological analysis. Scale bar, $200 \mu \mathrm{m}$ for tubular area and $50 \mu \mathrm{m}$ for glomerular area. (F and G) Glomerulosclerosis score and tubular injury score, respectively. (H) Transmission electron microscopy (scale bar, $500 \mathrm{~nm}$ ) and (I) podocyte injury score. NGAL, neutrophil gelatinase-associated lipocalin; BUN, blood urea nitrogen; DN, diabetic nephropathy.

A
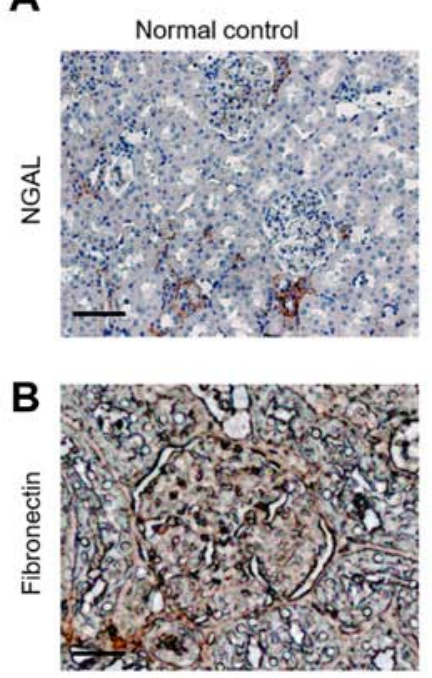

Glycemia
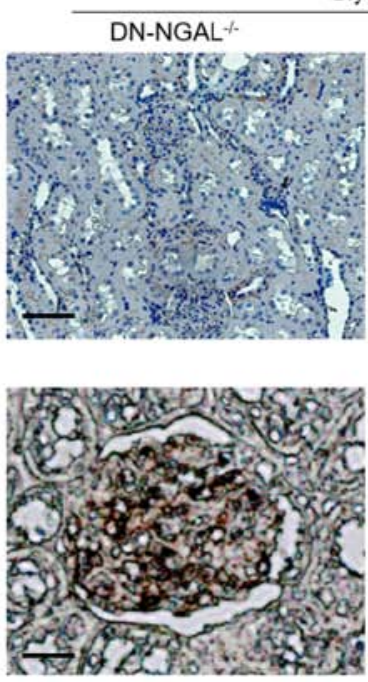
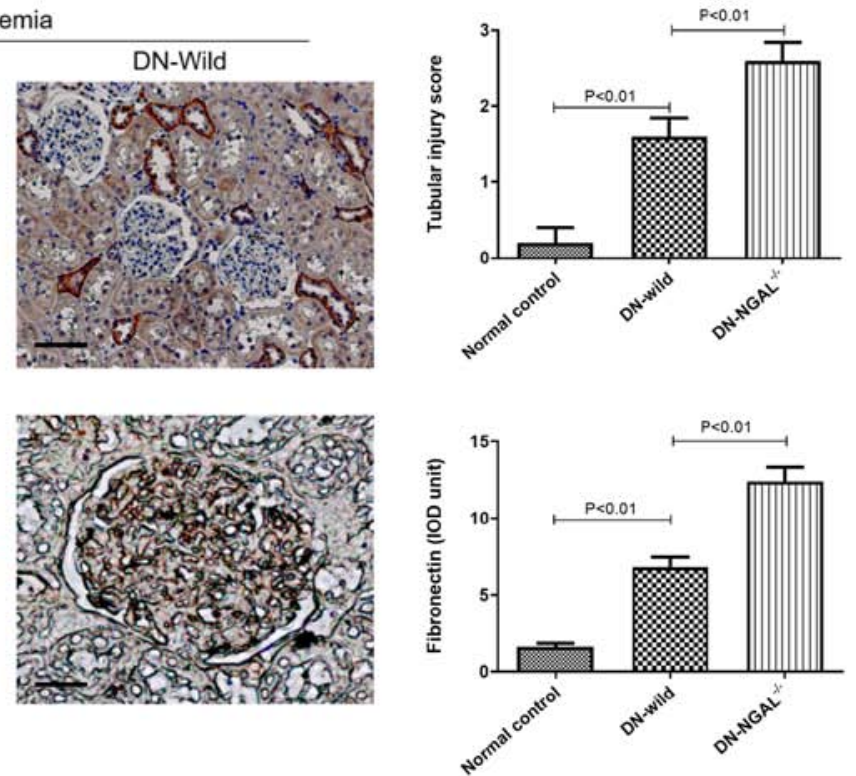

Figure 6. NGAL knockout exacerbates FN deposition in glomeruli in vivo, as analyzed by IHC. (A) NGAL expression in renal cortex, as detected by IHC, which confirmed that NGAL was not expressed in $\mathrm{NGAL}^{-/}$mice, scale bar, $200 \mu \mathrm{m}$. (B) FN deposit in glomeruli, as detected by IHC in the three groups, scale bar, $50 \mu \mathrm{m}$. IHC, immunohistochemistry; FN, fibronectin; NGAL, neutrophil gelatinase-associated lipocalin; DN, diabetic nephropathy.

\section{Discussion}

The majority of studies on NGAL have focused on its role in AKI, and NGAL seems to be a potential biomarker to predict kidney injury (33). However, its role in the pathophysiology and disease progression of chronic kidney dysfunctions, particularly DN, remains unclear. Since NGAL is mostly produced by proximal tubular epithelial cells, in the present study, the 
human tubular cell line HK2 was selected to investigate the effects of NGAL knockdown by RNA interference.

Oxidation plays a detrimental role in the progression of DN (34). In the current study, HG incubation caused higher ROS production in HK2 cells, and diabetic mice also showed higher oxidative stress in kidney tissues compared with that in normal mice. Flow cytometry suggested that NGAL significantly reduced ROS production under HG stimulation. Higher oxidative stress was also found in the kidney of $\mathrm{NGAL}^{-/}$mice compared with that of wild-type mice, evidence by the ROS, MDA, GSH, SOD and catalase results. Therefore, it is hypothesized that this is one of the mechanisms by which NGAL slows down the progression and deterioration of DN.

Chronic inflammation is another detrimental factor that can accelerate DN progression (35). NGAL is regarded an inflammatory mediator secreted by neutrophils to play a role in the early stage of acute inflammation (36). However, DN is associated with chronic inflammation; thus, the role of NGAL in the chronic inflammation of DN requires further investigation. In the current study, $\mathrm{HG}$ stimulation produced higher IL-6 levels in HK2 cells, and IL-6 has been demonstrated to be positively associated with DN progression and severity in a previous study (31). In addition, NGAL increased the production of IL-6 under HG stimulation. IL-6 is an important enhancer of chronic inflammation and its expression has been considered an important mediator of DN progression (37).

Previous studies have demonstrated that NGAL has a protective effect on experimental AKI, including LPS-induced AKI and cisplatin-induced AKI (38) by suppressing apoptosis via inhibition of caspase 3 activation (39). However, its role in DN remains unclear. The major characteristics of DN pathology include glomerulosclerosis, tubular epithelial cell necrosis and apoptosis, and the present study suggests that NGAL knockout may enhance these pathological injuries, and facilitate the progression of DN.

IHC of NGAL confirmed that diabetes could induce NGAL expression in the tubular area of kidney tissues, and also confirmed that NGAL was not expressed in knockout mice. Fibrosis is an important driving factor for glomerulosclerosis. In vitro, under HG and TGF- $\beta 1$ stimulation, HK2 cells with NGAL knockdown produced higher levels of extracellular matrix proteins, such as FN and Col IV, which are major components of the ECM. In vivo results also showed that knockout NGAL caused increased secretion of matrix proteins. Regarding the underlying mechanism of inhibiting fibrosis, the present study demonstrated that $\mathrm{NGAL}^{-/}$could promote Smad2/3 phosphorylation, which is considered a key process in the TGF- $\beta 1$ stimulation signaling pathway (40). The detailed mechanism by which NGAL cross talks with the TGF-Smad signaling pathway remains unclear and needs further study.

The limitation of the present study is that the mouse NGAL and the human NGAL sequences do not share close homogeneity; therefore, the bioactivities observed in mice may be different from those of humans.

In conclusion, by NGAL knockdown in vitro and by knockout in vivo, the renal protective effect of NGAL was confirmed under glycemia conditions. However, its detailed mechanism requires further investigation.

\section{Acknowledgements}

Not applicable.

\section{Funding}

Financial support was received from the Hebei Medical Research Key Subject Program for 2017 (grant no. 20160627).

\section{Availability of data and materials}

The datasets used and/or analyzed during the current study are available from the corresponding author on reasonable request.

\section{Authors' contributions}

$\mathrm{XL}$ and $\mathrm{XZ}$ designed all the experiments and carried out the experiments. XD performed the statistical analysis, and wrote the manuscript. XW and TW created the figures and helped with designing the experiments. SF, HZ, and CC assisted in experiments and manuscript writing. GL contributed to the conception of the study and manuscript revision. All authors read and approved the final version of the manuscript.

\section{Ethics approval and consent to participate}

The experimental protocol was approved by the Institutional Animal Ethics Committee of the Affiliated Hospital of Hebei University of Engineering (Handan, China) and performed in accordance with the guidelines on animal experimentation of the Committee for Control and Supervision of Experimentation on Animals, Government of China.

\section{Patient consent for publication}

Not applicable.

\section{Competing interests}

The authors declare that they have no competing interests.

\section{References}

1. Papadopoulou-Marketou N, Margeli A, Papassotiriou I, Chrousos GP, Kanaka-Gantenbein C and Wahlberg J: NGAL as an early predictive marker of diabetic nephropathy in children and young adults with type 1 diabetes mellitus. J Diabetes Res 2017: 7526919, 2017.

2. Sadar S, Kaspate D and Vyawahare N: Protective effect of L-glutamine against diabetes-induced nephropathy in experimental animal: Role of KIM-1, NGAL, TGF- $\beta 1$, and collagen-1. Ren Fail 38: 1483-1495, 2016.

3. Scelo G and Larose TL: Epidemiology and risk factors for kidney cancer. J Clin Oncol 36: JCO2018791905, 2018.

4. Tarchini R, Bottini E, Botti P, Talassi E, Baraldi O, Lambertini D, Gaetti L and Bellomi A: Type 2 diabetic nephropathy: Clinical course and prevention proposals 2004. G Ital Nefrol 22 (Suppl 31): S15-S19, 2005 (In Italian).

5. Leehey DJ, Singh AK, Alavi N and Singh R: Role of angiotensin II in diabetic nephropathy. Kidney Int (Suppl 77): S93-S98, 2000.

6. Zhang S, Wang D, Xue N, Lai F, Ji M, Jin J and Chen X: Nicousamide protects kidney podocyte by inhibiting the TGF $\beta$ receptor II phosphorylation and AGE-RAGE signaling. Am J Transl Res 9: 115-125, 2017. 
7. Zhang S, Li Y,Li H,Zheng X and Chen X: Renal-protective effect of nicousamide on hypertensive nephropathy in spontaneously hypertensive rats. Biomed Rep 1: 34-40, 2013.

8. Oh SM, Park G, Lee SH, Seo CS, Shin HK and Oh DS: Assessing the recovery from prerenal and renal acute kidney injury after treatment with single herbal medicine via activity of the biomarkers HMGB1, NGAL and KIM-1 in kidney proximal tubular cells treated by cisplatin with different doses and exposure times. BMC Complement Altern Med 17: 544, 2017.

9. Kaul A, Behera MR, Rai MK, Mishra P, Bhaduaria DS, Yadav S Agarwal V, Karoli R, Prasad N, Gupta A and Sharma RK: Neutrophil gelatinase-associated lipocalin: As a predictor of early diabetic nephropathy in type 2 diabetes mellitus. Indian J Nephrol 28: 53-60, 2018.

10. Cowland JB and Borregaard N: Molecular characterization and pattern of tissue expression of the gene for neutrophil gelatinase-associated lipocalin from humans. Genomics 45: 17-23, 1997.

11. Borregaard N, Sehested M, Nielsen BS, Sengeløv H and Kjeldsen L: Biosynthesis of granule proteins in normal human bone marrow cells. Gelatinase is a marker of terminal neutrophil differentiation. Blood 85: 812-817, 1995.

12. Flo TH, Smith KD, Sato S, Rodriguez DJ, Holmes MA, Strong RK, Akira S and Aderem A: Lipocalin 2 mediates an innate immune response to bacterial infection by sequestrating iron. Nature 432: 917-921, 2004.

13. Venge P, Douhan-Håkansson L, Garwicz D, Peterson C, Xu S and Pauksen K: Human neutrophil lipocalin as a superior diagnostic means to distinguish between acute bacterial and viral infections. Clin Vaccine Immunol 22: 1025-1032, 2015.

14. Mishra J, Mori K, Ma Q, Kelly C, Yang J, Mitsnefes M, Barasch J and Devarajan P: Amelioration of ischemic acute renal injury by neutrophil gelatinase-associated lipocalin. J Am Soc Nephrol 15: 3073-3082, 2004.

15. Mori K and Nakao K: Neutrophil gelatinase-associated lipocalin as the real-time indicator of active kidney damage. Kidney Int 71: 967-970, 2007.

16. Bolignano D, Coppolino G, Campo S, Aloisi C, Nicocia G, Frisina $\mathrm{N}$ and Buemi M: Urinary neutrophil gelatinase-associated lipocalin (NGAL) is associated with severity of renal disease in proteinuric patients. Nephrol Dial Transplant 23: 414-416, 2008.

17. Wang W, Li Z, Chen Y, Wu H, Zhang S and Chen X: Prediction value of serum NGAL in the diagnosis and prognosis of experimental acute and chronic kidney injuries. Biomolecules 10: 981, 2020.

18. Mauro C, Pacifico F, Lavorgna A, Mellone S, Iannetti A, Acquaviva R, Formisano S, Vito P and Leonardi A: ABIN-1 binds to NEMO/IKKgamma and co-operates with A20 in inhibiting NF-kappaB. J Biol Chem 281: 18482-18488, 2006.

19. Livak KJ and Schmittgen TD: Analysis of relative gene expression data using real-time quantitative PCR and the 2(-Delta Delta C(T)) method. Methods 25: 402-408, 2001.

20. Yan YM, Ai J, Zhou LL, Chung ACK, Li R, Nie J, Fang P, Wang XL, Luo J, Hu Q, et al: Lingzhiols, unprecedented rotary door-shaped meroterpenoids as potent and selective inhibitors of p-Smad3 from ganoderma lucidum. Org Lett 15: 5488-5491, 2013.

21. Zhang S, Ma J, Sheng L, Zhang D, Chen X, Yang J and Wang D: Total coumarins from Hydrangea paniculata show renal protective effects in lipopolysaccharide-induced acute kidney injury via anti-inflammatory and antioxidant activities. Front Pharmacol 8: 872, 2017.

22. Sen Z, Weida W, Jie M, Li S, Dongming Z and Xiaoguang C: Coumarin glycosides from Hydrangea paniculata slow down the progression of diabetic nephropathy by targeting Nrf2 anti-oxidation and smad2/3-mediated profibrosis. Phytomedicine 57: 385-395, 2019.
23. Nørgaard SA, Sand FW, Sørensen DB, Abelson KS and Søndergaard H: Softened food reduces weight loss in the streptozotocin-induced male mouse model of diabetic nephropathy. Lab Anim 52: 373-383, 2018.

24. National Technical Committee for Standardization of Laboratory Animals.

25. Naito T, Ma LJ, Yang H, Zuo Y, Tang Y, Han JY, Kon V and Fogo AB: Angiotensin type 2 receptor actions contribute to angiotensin type 1 receptor blocker effects on kidney fibrosis. Am J Physiol Renal Physiol 298: F683-F691, 2010.

26. Pörsti I, Fan M, Kööbi P, Jolma P, Kalliovalkama J, Vehmas TI, Helin H, Holthöfer H, Mervaala E, Nyman T and Tikkanen I: High calcium diet down-regulates kidney angiotensin-converting enzyme in experimental renal failure. Kidney Int 66: 2155-2166, 2004

27. Li P, Ma LL, Xie RJ, Xie YS, Wei RB, Yin M, Wang JZ and Chen XM: Treatment of 5/6 nephrectomy rats with sulodexide: A novel therapy for chronic renal failure. Acta Pharmacol Sin 33: 644-651, 2012.

28. Aunapuu M, Pechter U, Arend A, Suuroja T and Ots M: Ultrastructural changes in the remnant kidney (after $5 / 6$ nephrectomy) glomerulus after losartan and atenolol treatment. Medicina (Kaunas) 39: 975-979, 2003.

29. Fernandes SM, Cordeiro PM, da Fonseca CD and Vattimo MF: The role of oxidative stress in streptozotocin-induced diabetic nephropathy in rats. Arch Endocrinol Metab 60: 443-449, 2016.

30. Han H, Cao A, Wang L, Guo H, Zang Y, Li Z, Zhang X and Peng W: Huangqi decoction ameliorates streptozotocin-induced rat diabetic nephropathy through antioxidant and regulation of the TGF- $\beta$ /MAPK/PPAR- $\gamma$ signaling. Cell Physiol Biochem 42: 1934-1944, 2017.

31. Zhang S, Yang J, Li H, Li Y, Liu Y, Zhang D, Zhang F, Zhou W and Chen X: Skimmin, a coumarin, suppresses the streptozotocin-induced diabetic nephropathy in wistar rats. Eur J Pharmacol 692: 78-83, 2012.

32. Sen Z, Weida W, Li Y, Zhaojun L, Nina X and Xiaoguang C: Nicousamide attenuates renal dysfunction and glomerular injury in remnant kidneys by inhibiting TGF- $\beta 1$ internalisation and renin activity. Eur J Pharmacol 845: 74-84, 2019.

33. Han M, Li Y, Liu M, Li Y and Cong B: Renal neutrophil gelatinase associated lipocalin expression in lipopolysaccharide-induced acute kidney injury in the rat. BMC Nephrol 13: 25, 2012.

34. Papadopoulou-Marketou N, Paschou SA, Marketos N, Adamidi S, Adamidis S, and Kannaka-Gantenbein C: Diabetic nephropathy in type 1 diabetes. Minerva Med 109: 218-228, 2018.

35. Pérez-Morales RE, Del Pino MD, Valdivielso JM, Ortiz A, Mora-Fernández $\mathrm{C}$ and Navarro-González: Inflammation in diabetic kidney diseases. Nephron 143: 12-16, 2019.

36. Shang W and Wang Z: The update of NGAL in acute kidney injury. Curr Protein Pept Sci 18: 1211-1217, 2017.

37. Feigerlová E and Battaglia-Hsu SF: IL-6 signaling in diabetic nephropathy: From pathophysiology to therapeutic perspectives. Cytokine Growth Factor Rev 37: 57-65, 2017.

38. Ma Q, Devarajan SR and Devarajan P: Amelioration of cisplatin-induced acute kidney injury by recombinant neutrophil gelatinase-associated lipocalin. Ren Fail 38: 1476-1482, 2016

39. Han M, Li Y, Wen D, Liu M, Ma Y and Cong B: NGAL protects against endotoxin-induced renal tubular cell damage by suppressing apoptosis. BMC Nephrol 19: 168, 2018.

40. Higgins SP, Tang Y, Higgins CE, Mian B, Zhang W, Czekay RP, Samarakoon R, Conti DJ and Higgins PJ: TGF- $\beta 1 / \mathrm{p} 53$ signaling in renal fibrogenesis. Cell Signal 43: 1-10, 2018.

This work is licensed under a Creative Commons Attribution-NonCommercial-NoDerivatives 4.0 International (CC BY-NC-ND 4.0) License. 